\title{
The effect of amino acid deletions and substitutions in the longest loop of GFP
}

\author{
Gabriela Flores-Ramírez ${ }^{\dagger}$, Manuel Rivera ${ }^{\dagger}$, Alfredo Morales-Pablos, \\ Joel Osuna, Xavier Soberón and Paul Gaytán*
}

\begin{abstract}
Address: Departamento de Ingeniería Celular y Biocatálisis. Instituto de Biotecnología, Universidad Nacional Autónoma de México, Ap. Postal 510-3 Cuernavaca, Morelos 62250, México

Email: Gabriela Flores-Ramírez - gabyflo32@hotmail.com; Manuel Rivera - manuel@ibt.unam.mx; Alfredo Morales-

Pablos - morapab@ibt.unam.mx; Joel Osuna - joel@ibt.unam.mx; Xavier Soberón - soberon@ibt.unam.mx; Paul Gaytán* - paul@ibt.unam.mx

* Corresponding author †Equal contributors
\end{abstract}

Published: 26 June 2007

BMC Chemical Biology 2007, 7:1 doi:10.1 186/1472-6769-7-1

This article is available from: http://www.biomedcentral.com//472-6769/7/I

(c) 2007 Flores-Ramírez et al; licensee BioMed Central Ltd.

This is an Open Access article distributed under the terms of the Creative Commons Attribution License (http://creativecommons.org/licenses/by/2.0), which permits unrestricted use, distribution, and reproduction in any medium, provided the original work is properly cited.
Received: I5 February 2007

Accepted: 26 June 2007

\begin{abstract}
Background: The effect of single and multiple amino acid substitutions in the green fluorescent protein (GFP) from Aequorea victoria has been extensively explored, yielding several proteins of diverse spectral properties. However, the role of amino acid deletions in this protein -as with most proteins- is still unknown, due to the technical difficulties involved in generating combinatorial inphase amino acid deletions on a target region.
\end{abstract}

Results: In this study, the region II 29-LI 42 of superglo GFP (sgGFP), corresponding to the longest loop of the protein and located far away from the central chromophore, was subjected to a random amino acid deletion approach, employing an in-house recently developed mutagenesis method termed Codon-Based Random Deletion (COBARDE). Only two mutants out of 16384 possible variant proteins retained fluorescence: sgGFP- $\Delta$ II29 and sgGFP- $\triangle$ DI30. Interestingly, both mutants were thermosensitive and at $30^{\circ} \mathrm{C}$ sgGFP- $\Delta$ DI 30 was more fluorescent than the parent protein. In contrast with deletions, substitutions of single amino acids from residues $\mathrm{FI} 3 \mathrm{I}$ to $\mathrm{LI} 42$ were well tolerated. The substitution analysis revealed a particular importance of residues $\mathrm{FI} 3 \mathrm{I}$, GI35, II37, LI38, HI40 and LI 42 for the stability of the protein.

Conclusion: The behavior of GFP variants with both amino acid deletions and substitutions demonstrate that this loop is playing an important structural role in GFP folding. Some of the amino acids which tolerated any substitution but no deletion are simply acting as "spacers" to localize important residues in the protein structure.

\section{Background}

The green fluorescent protein (GFP) has revolutionized molecular and cell biology, because it can be used as a reporter of gene expression and protein localization due to its inherent capacity to generate an efficiently emitting internal fluorophore [1-3]. GFP is a $28 \mathrm{kDa}$ protein com- posed of 238 amino acid residues. X-ray crystal studies of GFP uncovered a $\beta$-barrel structure resembling a soda can. The wall of the $\beta$-can structure is built by 11 antiparallel $\beta$-strands. This $\beta$-sheet secondary structure surrounds a single central $\alpha$-helix that contains the fluorophore, spontaneously formed by post-translational modification of 
residues Ser65, Tyr66 and Gly67. Two protein lids, composed mainly of residues 74-91 and 128-145, cover the $\beta$-can structure and isolate the chromophore from the surrounding solvent. Because of the simplicity of the chromophore formation, modifications on the primary structure of GFP have produced several improved variants, either more fluorescent [4] or blue/red-shifted [5]. These changes have been achieved employing site-directed approaches [6], regional combinatorial approaches [4] and fully random approaches such as DNA shuffling [7].

High resistance to proteolysis [3], detergents [8], heat [9] and denaturing agents [9] are consequences of the rigid structure of GFP which seems to be a nearly size-minimized protein [10]. GFP tolerates enlargement through the insertion of short peptides [11], long peptides [12] and even complete proteins [13] in different locations, but it is particularly sensitive to shortening by internal site-directed deletions $[10,11]$. Prior to this study, only amino- or carboxyl-terminus deletions have been reported for GFP [10].

The role of insertions and deletions (indels) in protein evolution is likely to be very significant, as can be inferred by inspecting any sequence or structure alignment of homologous proteins. Unfortunately, their role has been difficult to assess experimentally, due to lack of convenient methods to generate indels systematically. Recently, we described a novel and unique mutagenesis method (named COBARDE) capable to generate codon-based random amino acid deletions on interesting protein regions [14]. Using COBARDE, the specificity of the enzyme TEM$1 \beta$-lactamase was modified by random combination of several amino acid deletions located around the active site.

To extend the evaluation of COBARDE as a potential tool in protein engineering, particularly to explore the relationship between protein size and function, a systematic search of deletions in the region 129-142 of superglo Green Fluorescent Protein (sgGFP) was undertaken in the present study. This region is equivalent to amino acids 128-141 of wild-type GFP from Aequorea victoria and corresponds to the longest loop of the protein (see Fig. 1).

\section{Results and discussion}

COBARDE was originally tested on TEM- $1 \beta$-lactamase with interesting results [14]. There were clear indications that this enzyme was able to tolerate even long internal deletions [15] and this was confirmed by the systematic introduction of deletions. GFP is, however, completely different because no active internal deletions have been yet reported. We thought an excellent test bed for COBARDE was to attempt to shorten this already rather rigid and structurally compromised protein.
We selected the region located between residues 129-142 as target of the mutagenesis for three reasons: 1) It is the longest loop of the protein; 2) two previous attempts of deletions in this area failed to produce fluorescent proteins $[10,11]$; 3) Published sequence alignments of GFP versus GFP-like proteins of anthozoas suggest that GFP may tolerate deletion of either G138 [16] or H139 [17] (G139 and H140 respectively in sgGFP).

Experimental work started with synthesis of the oligonuclotide library. One current limitation of Fmoc-based mutagenesis methods is depurination of benzoylated deoxyadenosines $\left(\mathrm{dA}^{\mathrm{bz}} \mathrm{s}\right)$, giving rise to a high ratio of backbone cleavage (our own unpublished results). This depurination problem is magnified if the target sequence is dA-enriched at the $3^{\prime}$ end, because synthesis proceeds from 3' towards 5' direction. The severity of the problem prevented a successful synthesis of the coding strand for the targeted region. Thus, we resorted to synthesizing a complementary sequence, further modified to reduce even more the content of $\mathrm{dA}^{\mathrm{bz}} \mathrm{s}$ near the 3 'end (indicated in bold face): $3^{\prime}$ ctc gac ttt cca TAG CTG AAG TTC CTT CTG CCG TTG TAG GAC CCT GTG TTT GAC ctt atg ttg ata $\operatorname{ttg} 5^{\prime}$. This sequence contains 17 fewer $\mathrm{dA}^{\mathrm{bz}}$ s than the original sequence and was successfully assembled by COBARDE. The oligonucleotide was used as a PCR template of two partially complementary primers to generate a 148 bp double stranded fragment that included the Mlu I and Acc I restriction sites as shown in Figure 2. The product was digested, and ligated to the kanamycin-carrying cloning vector pT4GFPMlu (see M\&M for preparation of this recipient plasmid). The ligation mixture was transformed into XL1-Blue cells to give a library of $2 \times 10^{6}$ variants. Analysis of colonies grown on plates for $24 \mathrm{~h}$ at $37^{\circ} \mathrm{C}$ revealed that more than $99 \%$ of the transformants were non-fluorescent to the naked eye, indicating that most of the deletions perturbed protein structure and/or function. Plasmid DNA from 40 randomly chosen fluorescent clones was obtained and sequenced revealing that 14 of the samples corresponded to re-ligated vector due to incomplete Mlu I/Acc I digestion; 22 corresponded to wild-type sgGFP created with the wild-type oligonucleotide generated in the library and only 4 of the clones were mutants that retained fluorescence. These mutants corresponded to single amino acid deletions of isoleucine 129 (sgGFP- $\Delta$ I129) and aspartate 130 (sgGFP- $\Delta$ D130). Each mutant was found twice.

On the other hand, the DNA sequence analysis of 33 nonfluorescent colonies (Table 1) gave an estimation of the quality of the library and provided insights into the kind of mutations that destroy fluorescence. From the data shown in Table 1 we draw the following conclusions: 


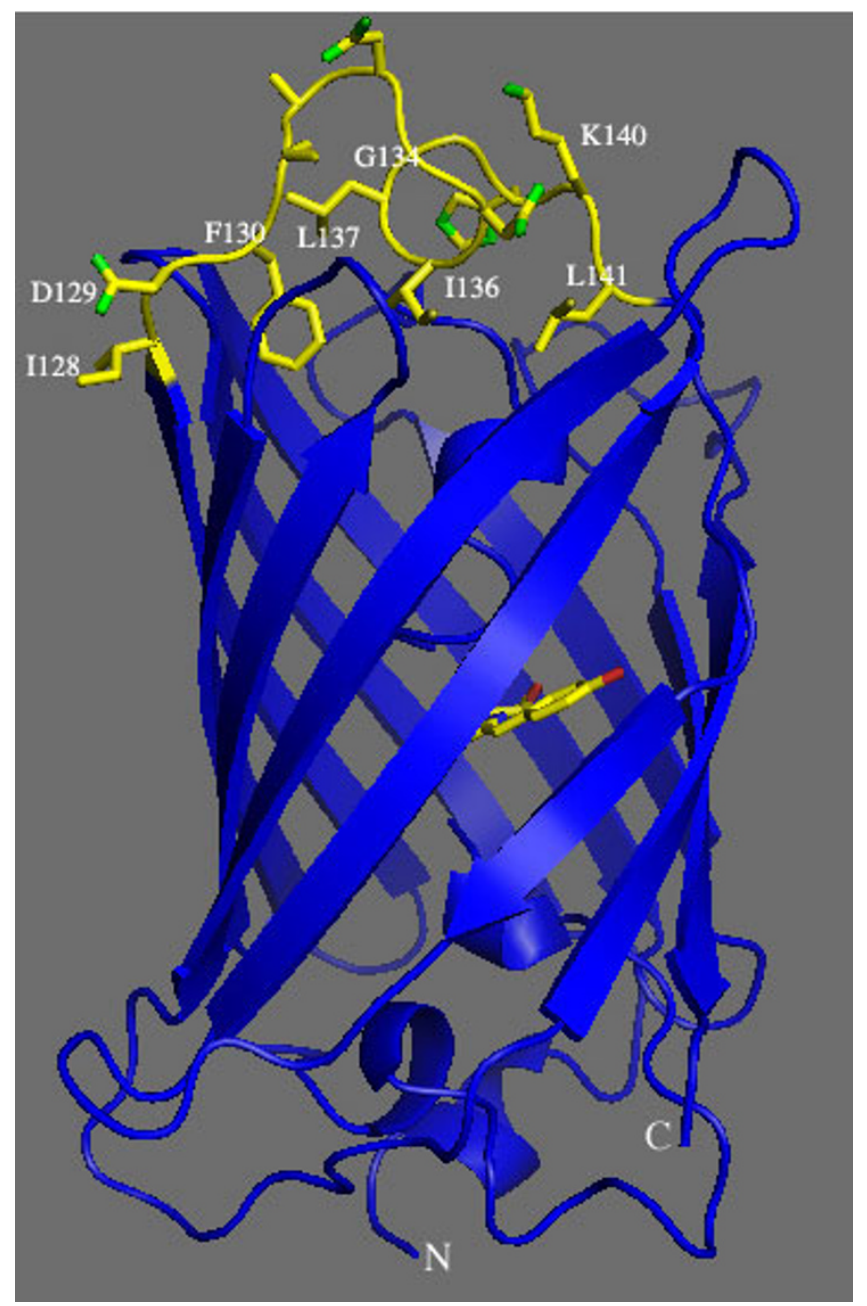

Figure I

Structure of wild-type GFP (IEMA). The region I28I4 | (129-142 in sgGFP) explored in this study is shown in yellow. $\mathrm{N}$-terminal and $\mathrm{C}$-terminal are marked with capital letters and the chromophore located in the center of the barrel is also shown in yellow.

1) A successful mutagenesis (with an average $50 \%$ mutagenesis rate) was achieved on the target region. It is clear from Table 1 that amino acid deletions were well spread and represented along the target, except for the first codon (encoding I129) which was mutated at only $2 \%$ rate because the Fmoc-Cl delivering line was not properly primed. However, this failure was corrected from the second codon on.

2) 6 out of 33 clones (clones 28-33) contained either single nucleotide deletions or insertions that change the open reading frame of the genes. Although this ratio of undesired variants is apparently high $(18 \%)$, it is within the error range found in conventional oligonucleotides as has been observed during assembly of synthetic genes [18-
20]. Single nucleotide deletions usually occur because of incomplete capping step during each synthesis cycle. This chemical imperfection may be significantly reduced with the use of UNICAP [21], a recently commercially available potent capping reagent. However, the remaining $1.68 \times$ $10^{6}$ useful variants $(82 \%)$ are enough to represent the complete set of $16384\left(2^{14}\right)$ possible deletion variants. Considering an average 0.5 mutagenesis rate per codon, each of the mutants should be represented with the same frequency and we only need a library of 75492 clones to find the least represented variant with 99\% confidence [22]. Further, since the wild-type clone was found several times in the fluorescence screening, it can be concluded that all mutants were well represented in the experimental library.

3) The library follows a roughly binomial distribution. Mutants carrying 6, 7, 8 and 9 deletions were the most frequent.

4) Most of the deletions in the explored loop destroy GFP fluorescence. This result agrees with those found by Li et al [10] and Kitamura et al [11]. Li removed the region comprising amino acids 132-139 of GFP by site-directed mutagenesis, whereas Kitamura randomly removed tripeptide blocks in the region 125-142. Both studies found the deletions to cause a complete loss of fluorescence.

Our sample of 33 non-fluorescent mutants sequenced included only one single deletion mutant, sgGFP- $\Delta$ K141, yet two single deletions, sgGFP- $\Delta$ I129 and sgGFP- $\Delta$ D130, conserved fluorescence. To make sure that our fluorescence screening was able to pick up all active robust mutants, we decided to individually create the remaining 11 single deletion mutants and the double mutant that combines $\Delta \mathrm{I} 129$ and $\Delta \mathrm{D} 130$ by site-directed mutagenesis using the specific oligonucleotides shown on Figure 2.

Confirming the validity of the library screening, none of the E. coli expressing these mutants displayed a green-fluorescent phenotype on plates, after incubation at $37^{\circ} \mathrm{C}$ for $24 \mathrm{~h}$. Fluorescence scanning of cultures containing each of the fourteen single deletion mutants and the double mutant, grown for $12 \mathrm{~h}$ at $37^{\circ} \mathrm{C}$, confirmed the results observed in plates. These experiments also discarded the hypothesis that sgGFP- $\Delta$ G139 and sgGFP- $\Delta H 140$ may be functional, as suggested by the alignments of GFP versus GFP-like proteins.

Other aligments based in three-dimensional structures of GFP versus GFP-like proteins suggest that region 128-141 does not tolerate deletions and that GFP must tolerate deletion of Y143 $[23,24]$. To test the confidence of these $3 \mathrm{D}$ aligments for protein engineering, we removed the equivalent residue (Y144) in sgGFP by site-directed muta- 


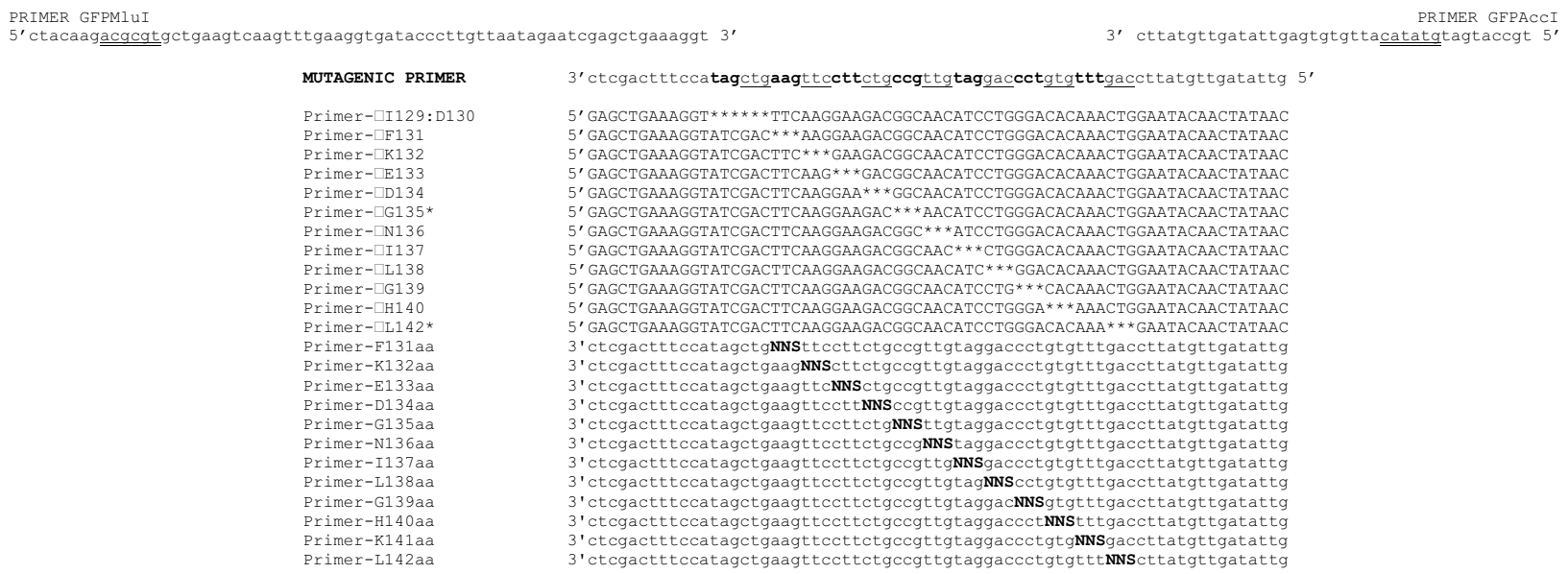

Figure 2

Oligonucleotides used for preparation of sgGFP mutants as described in M\&M. For clear visualization, codons subjected to combinatorial deletion are alternatively labeled in bold and underlined. Asterisks represent nucleotide deletions. N represents an equimolar mixture of the four nucleotides and $\mathrm{S}$ a mixture of $\mathrm{G}$ and $\mathrm{C}$. Restriction sites are double-underlined.

genesis and the fluorescence was completely lost. The conclusion of these aligments is obvious, no prediction can be done when the sequence identity between the proteins compaired is so low. The sequence identity between GFPs and GFP-like proteins is around $25 \%$.

Additional characterization of whole cells containing the mutants sgGFP- $\Delta$ I129 and sgGFP- $\Delta$ D130 revealed that both proteins suffered a blue-shift of two nanometers in their maximum emission and their fluorescence intensity was reduced to $21 \%$ and $17 \%$, respectively, relative to wt sgGFP. The last result did not correlate with the phenotype observed in plates, where the green color of the mutants was only slightly less intense than the wild-type protein. We then decided to measure the quantum yield of the mutant proteins, which turned out to be $31 \%$ and $21 \%$ smaller than the parent protein, respectively. Because the quantum yield decrement of the mutants did not fully account for the fluorescence loss, we turned our attention towards protein concentration in the cells, another factor that affects fluorescence intensity. The amount of soluble and non-soluble protein for each mutant was analyzed by western blotting as shown in Figure 3, using anti-GFP for the detection. This experiment clearly revealed that the main reason for the reduction or loss of fluorescence of the mutants was their low concentration which, in turn, could also be due to low stability or incorrect folding [25]. Not surprisingly, sgGFP- $\Delta$ I129 and sgGFP- $\Delta$ D130 were the best mutants expressed. To assess if the proteins were inactivated by improper folding we grew the mutants at $30^{\circ} \mathrm{C}$. At this lower temperature, the fluorescence of sgGFP- $\triangle$ I129 increased from $21 \%$ to $46 \%$, whereas sgGFP- $\triangle$ D 130 increased from $17 \%$ to $116 \%$ as compared to wt sgGFP. These results indicated that both deletion mutants are thermosensitive, and even more, at lower temperatures sgGFP- $\Delta$ D130 is more fluorescent than the wild-type protein. Lower temperatures frequently favor appropriate folding of mutants [13]. Western blotting of the mutants grown at $30^{\circ} \mathrm{C}$, shown on Figure $3 \mathrm{~b}$, confirmed that the protein concentration was increased.

It is worth mentioning that plated colonies expressing the other single deletion mutants remained being non-fluorescent neither at $30^{\circ} \mathrm{C}$ nor at $22^{\circ} \mathrm{C}$ during 15 days of growing.

Temperature denaturation curves (see Figure 4) for the active purified mutants sgGFP- $\Delta$ I129 and sgGFP- $\Delta$ D 130 , demonstrated that these proteins are less heat stable than the parent protein, but not enough to give account for the significative protein reduction at $37^{\circ} \mathrm{C}$. Therefore, these amino acids are essential for good folding, especially D130.

In the case of some non-functional mutants such as sgGFP- $\Delta$ L138 and sgGFP- $\Delta I 129 / \Delta$ D130, low protein concentration was not the only explanation for their loss of fluorescence. These two mutants gave rise to significant inclusion bodies but still a considerable amount of protein remained in solution, which would be expected to 
Table I: DNA sequence of non-fluorescent clones chosen randomly from the library generated with COBARDE

\begin{tabular}{|c|c|c|c|c|c|c|c|c|c|c|c|c|c|c|c|}
\hline \multirow[t]{2}{*}{ Clone } & \multicolumn{14}{|c|}{ sgGFP modified region } & \multirow[t]{2}{*}{ Codons deleted } \\
\hline & Ile 129 & Asp I30 & Phe $13 \mid$ & Lys 132 & Glu 133 & Asp 134 & Gly 135 & Asn 136 & Ile 137 & Leu 138 & Gly 139 & His 140 & Lys 141 & Leu 142 & \\
\hline Wt & & & & & & & & & & & & & & & 0 \\
\hline 1 & ATC & & TTC & & & & GGC & AAC & & & & & & & 10 \\
\hline 2 & ATC & GAC & TTC & AAG & GAA & GAC & GGC & AAC & ATC & CTG & GGA & CAC & & CTG & 1 \\
\hline 3 & ATC & GAC & TTC & & & & & AAC & & & GGA & CAC & AAA & CTG & 6 \\
\hline 4 & ATC & & TTC & AAG & & & & AAC & ATC & CTG & GGA & CAC & & CTG & 5 \\
\hline 5 & ATC & GAC & TTC & AAG & GAA & GAC & GGC & AAC & & CTG & & & & & 5 \\
\hline 6 & ATC & & & & & & & AAC & ATC & & GGA & CAC & & CTG & 8 \\
\hline 7 & ATC & & TTC & & & GAG & GGC & & & & & & & CTG & 9 \\
\hline 8 & ATC & GAC & & & & GAC & & $\mathrm{AAC}$ & & CTG & & & & & 9 \\
\hline 9 & ATC & GAC & & AAG & GAA & GAC & GGC & AAC & & & GGA & & AAA & CTG & 4 \\
\hline 10 & ATC & GAC & TTC & AAG & & & & AAC & & CTG & GGA & & & CTG & 6 \\
\hline 11 & ATC & GAC & & & GAA & GAC & & & ATC & & & & & & 9 \\
\hline 12 & ATC & GAC & & & & GAC & GGC & & ATC & CTG & GGA & & & & 7 \\
\hline 13 & ATC & GAC & TTC & AAG & GAA & GAC & GGC & AAC & & & & CAC & & CTG & 4 \\
\hline 14 & ATC & GAC & & AAG & GAA & & GGC & AAC & ATC & CTG & & CAC & AAA & CTG & 3 \\
\hline 15 & ATC & GAC & & AAG & GAA & GAC & & & & CTG & & & & & 8 \\
\hline 16 & ATC & GAC & & & GAA & GAC & GGC & & ATC & & & CAC & & CTG & 6 \\
\hline 17 & ATC & GAC & & AAG & GAA & GAC & & AAC & & & & & & & 8 \\
\hline 18 & ATC & GAC & & & & & & AAC & & & & & AAA & CTG & 9 \\
\hline 19 & ATC & GAC & TTC & & & GAC & GGC & & ATC & CTG & GGA & & AAA & CTG & 4 \\
\hline 20 & ATC & GAC & TTC & AAG & & GAC & & & & & & & & CTG & 8 \\
\hline 21 & ATC & GAC & TTC & & GAA & GAC & GGC & $\mathrm{AAC}$ & & & GGA & CAC & AAA & CTG & 3 \\
\hline 22 & ATC & GAC & TTC & & & & & AAC & & CTG & & & AAA & CTG & 7 \\
\hline 23 & ATC & & & AAG & & GAC & & & & CTG & & & & & 10 \\
\hline 24 & ATC & & TTC & AAG & & & GGC & AAC & & & & & & & 9 \\
\hline 25 & ATC & & TTC & & GAA & & GGC & & ATC & CTG & & & & CTG & 7 \\
\hline 26 & ATC & GAC & TTC & & GAA & GAC & GGC & AAC & & & GGA & CAC & AAA & CTG & 3 \\
\hline 27 & ATC & GAC & TTC & & GAA & & GGC & & & CTG & & & & & 8 \\
\hline 28 & ATC & G & TTC & & GAA & & GGC & $\mathrm{AAC}$ & & CTG & & CAC & & CTG & 6 \\
\hline 29 & ATC & GAC & & AAG & & & GGC & AAC & ATC & & GGA & & & $\mathrm{CT}$ & 6 \\
\hline 30 & ATC & & $T$ & AAG & & GAC & & $\mathrm{AAC}$ & & CTG & GGA & & AAA & CTG & 5 \\
\hline 31 & $A C$ & GAC & & & & & & & ATC & CTG & GGA & CAC & AAA & CTG & 6 \\
\hline 32 & ATC & & & & & $\mathrm{GA}$ & GGC & $\mathrm{AAC}$ & & CTG & GGA & & AAA & & 7 \\
\hline 33 & ATC & GAC & & AAG & & & & AAC & & CTG & $\mathrm{GA}$ & & & & 8 \\
\hline oligo & TAG & CTG & AAG & TTC & CTT & CTG & CCG & TTG & TAG & GAC & СCT & GTG & TTT & GAC & \\
\hline
\end{tabular}

Empty spaces represent deleted codons. Last row contains the oligonucleotide segment that was subjected to mutagenesis. Dots represent single nucleotide deletions. Nucleotide insertions are shown in gray letters.

give a signal if the proteins were fluorescent per se. We believe these mutants are correctly folded but maturation of the chromophore is impaired, in a mode similar to the colorless GFP isolated from Aequorea corulescens (acGFP) or the enhanced mutant aceGFP-G222E [26]. More biophysical and biochemical assays are needed to elucidate which process(es) are affected - cyclization, oxidation or dehydration.

The most important conclusion resulting from the deletion studies reported hereby is the key role of residues 131-142 (130-141 in GFP) for appropriate folding of the protein. This result agrees with results reported by Baird $e t$ al [13] working with permutations. They found that GFP can be opened in different locations only after residue N144 but they did not explain the absence of openings in the first half of the protein. Therefore, the region 131-142 seems to be acting as a bridge that joins two parts of the protein independently folded.
To further explore how essential is the sequence at the sub-region 131-142 we decided to subject each of the twelve positions to single site-saturation mutagenesis (see $M \& M$ for details) using the degenerated oligonucleotides shown on Figure 2. To our surprise, most of the variants $(55 \%)$ found in the libraries of substitutions displayed a green-fluorescent phenotype in plates, after $24 \mathrm{~h}$ of growth at $37^{\circ} \mathrm{C}$, showing that substitutions are tolerated where deletions are not. DNA sequence data from several randomly chosen fluorescent and non-fluorescent colonies (as appeared in the plate assay) are concentrated in Table 2. The data show that G135 is the least tolerant amino acid, with allowed replacements of this residue only producing pale green-fluorescent colonies (due to diminished soluble protein in the cells; data not shown). This buried amino acid forms part of a short $\alpha$-helix located at the center of the loop. Apparently, the major function of this $\alpha$-helix is to position I137 towards the heart of the barrel in order to fix part of the loop. 
A)
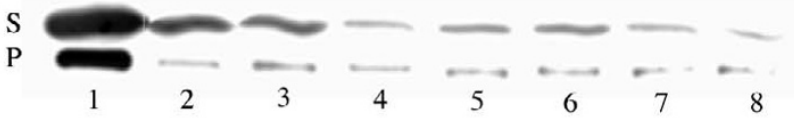

9

10

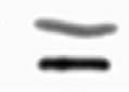

11

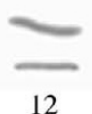

12

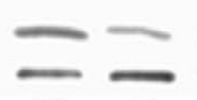

13

14

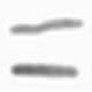

15

B)

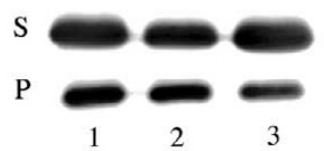

Figure 3

Western blotting analysis of deleted mutants grown at two different temperatures. (A) Single amino acid deletions comprised in the region I29-I42 of sgGFP and the double mutant sgGFP- $\Delta I / 29 / \Delta \mathrm{DI} 30$ grown at $37^{\circ} \mathrm{C}$. (B) Some sgGFP mutants grown at $30^{\circ} \mathrm{C}$. $S$ and $P$ represent the soluble and insoluble fraction of the cells, respectively. Fractions $S$ and $P$ were run on different gels. Lane I: sgGFP wt, lane 2: sgGFP- $\Delta$ II29, lane 3: sgGFP- $\triangle D \mid 30$, lane 4: sgGFP- $\Delta F \mid 3$ I, lane 5: sgGFP- $\Delta \mathrm{K} I 32$, lane 6: sgGFP- $\Delta$ EI33, lane 7: sgGFP- $\Delta$ D I34, lane 8: sgGFP- $\Delta$ GI35, lane 9: sgGFP- $\Delta$ NI 36, lane I0: sgGFP- $\Delta$ II 37, lane II: sgGFP$\Delta \mathrm{LI}$ 38, lane I2: sgGFP- $\Delta$ G I39, lane I3: sgGFP- $\Delta$ HI40, lane I4: sgGFP- $\Delta$ K I4I, lane I5: sgGFP- $\Delta$ LI 42 and lane I6: sgGFP- $\Delta$ II29/ $\Delta \mathrm{DI} 30$.

Positions $131,137,138$ and 142 only tolerated conservative replacements with hydrophobic residues. Because F131, L138 and L142 are also buried in the core of the protein, these amino acids are likely to be important for fixation of the loop. The non-fluorescent mutant F130A also revealed that size of the hydrophobic side-chain is important for appropriate packaging of the protein. On the other hand, residues H140 and K141 were replaced only with hydrophilic amino acids, suggesting ionic or $\mathrm{H}$ bond interactions with neighbor amino acids. Finally, residues K132, E133, D134, N136 and G139, whose sidechains are exposed to the solvent, tolerated any amino acid substitution.

Our results with substitutions confirmed the scope of the scanning mutagenesis approach to identify buried and exposed amino acids in proteins of unknown structure as proposed by Bajaj et al [27]. For instance, substitution of buried amino acids with charged residues, as in mutants F131D, F131K, I137D, I137E, I137R and L142D, destroyed fluorescence, apparently because of protein instability. However, it is important to note that some mutants (labeled with asterisk in Table 2), initially nonfluorescent in the plate assay, turned pale green-fluorescent after 3-5 additional days of growing at room temperature, suggesting slow maturation of the chromophore as in the case of acGFP [26].

\section{Conclusion}

COBARDE has been demonstrated to be a powerful and confident mutagenesis tool to reduce, although to a minimal amount, a particularly constrained structure and rigid protein such as GFP. The method allowed us to select two unique active mutants out of 16384 possible variant proteins in a stretch of 14 amino acids. If this achievement is extrapolated to enzymes, then the optimal mutant could be easily obtained using appropriate selection conditions. The radical difference between the complete exploration of random deletions versus random substitutions is the library size. For instance, if the same stretch of 14 amino acids is subjected to substitution, using a mixture of 20 trimers to saturate each of the wild-type codons, a library of $1.6 \times 10^{18}\left(20^{14}\right)$ variants would be generated. The complete transformation of this amount is impossible to achieve and, consequently, one would never be sure if the best mutant has been expressed and selected in the biological libraries. Even more, COBARDE might be easily extended to explore regions up to 18 amino acids with a practical transformation efficiency of $10^{7}$ variants.

On the other hand, the systematic generation of single amino acid deletions and single substitutions on the region 129-142 of sgGFP demonstrated that some residues, highly tolerant to substitutions but intolerant to deletions, play a simple "spacer" role to locate near residues in appropriate positions of the protein. It also confirmed that deletions are more disruptive events than substitutions, affecting mainly the protein folding and stability. Knowing these apparently adverse results, the question is obvious: is it really worth studying deletions? The answer is clearly YES. COBARDE might be used to reduce the size of several therapeutic proteins in order to modify their diffusion in the body and might also be used to shorten or enlarge the active site of enzymes (depending on the region subjected to mutagenesis) in order to accept smaller or larger substrates than the natural ones. 


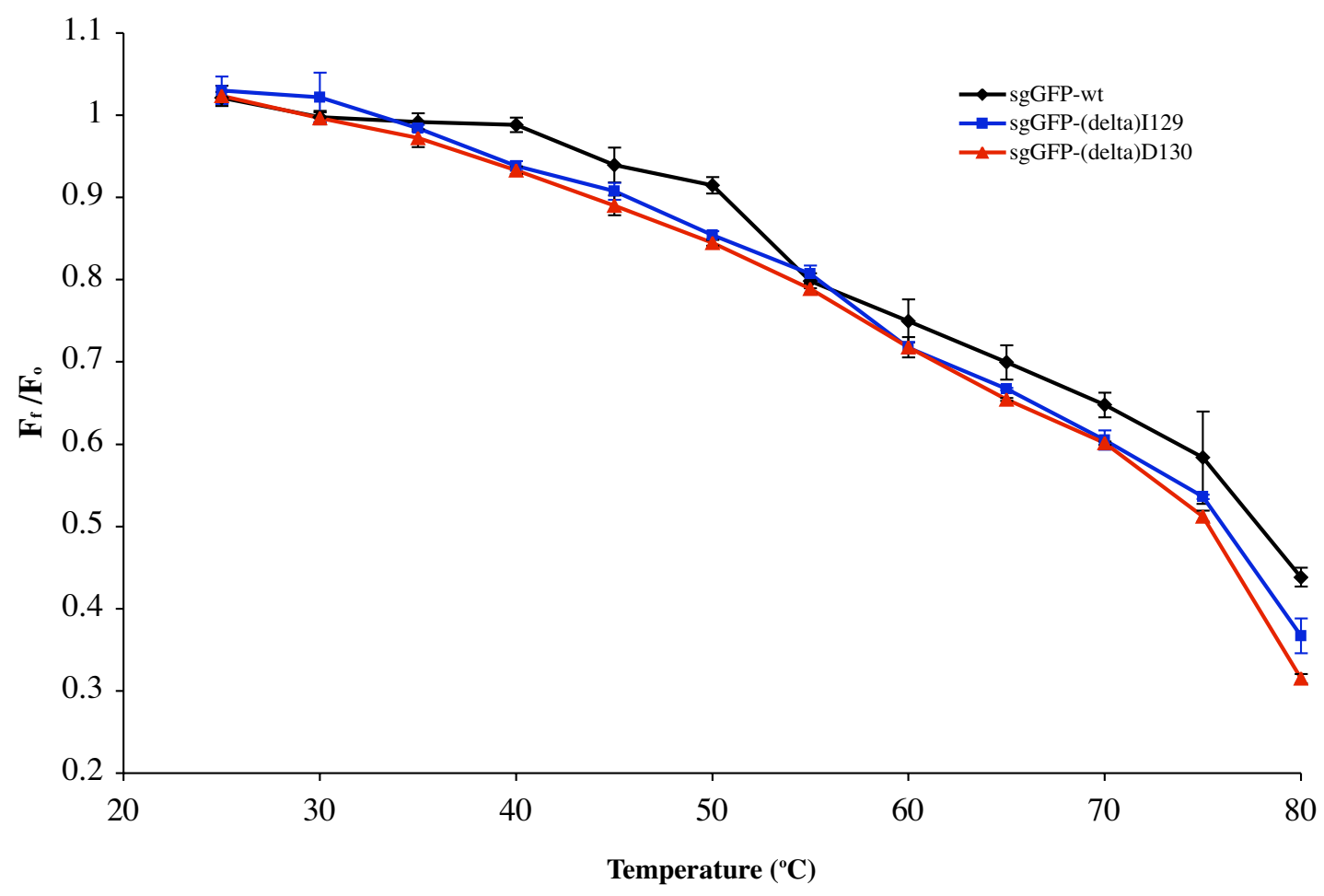

\section{Figure 4}

"Temperature denaturation curves of the purified proteins sgGFP wt, sgGFP- $\Delta I \mid 29$ and sgGFP- $\Delta$ DI 30 . Triplicated aliquots of each sample were subjected to different temperatures $\left(25^{\circ} \mathrm{C}, 30^{\circ} \mathrm{C}, 35^{\circ} \mathrm{C}, 40^{\circ} \mathrm{C}, 45^{\circ} \mathrm{C}, 50^{\circ} \mathrm{C}, 55^{\circ} \mathrm{C}, 60^{\circ} \mathrm{C}\right.$, $65^{\circ} \mathrm{C}, 70^{\circ} \mathrm{C}, 75^{\circ} \mathrm{C}$ and $\left.80^{\circ} \mathrm{C}\right)$ during 5 minutes and the initial $\left(F_{o}\right)$ and final fluorescence $\left(F_{f}\right)$ was measured. The ratio $F_{f} / F_{o}$ versus temperature was plotted."

\section{Methods}

\section{Oligonucleotide synthesis}

The Codon-Based Random Deletion (COBARDE) method relies on oligonucleotide synthesis. Briefly, COBARDE consists of arresting part of the growing oligo in the preceding nucleotide to the target codon to be deleted. The partial arresting is performed by substoichiometric reaction of the growing oligo with a diluted solution of fluorenylmetoxycarbonyl-chloride (Fmoc-Cl), in the presence of 4,4-dimethylaminopiridine (DMAP) as catalyst. The Fmoc protecting group is known to be stable to acid and labile to alkali. In the following step, the wildtype codon is assembled by performing three consecutive couplings of dimethoxytrityl (DMTr) protected monomers with the unblocked oligo. Both, Fmoc and DMTr groups are subsequently removed using alkali and acid treatment respectively, and the growing oligo is ready for another deletion cycle. If the substoichiometric reaction is high, the library will be enriched with mutants carrying several codon deletions, but if the substoichiometric reaction is low the library will be enriched with mutants carrying few codon deletions [14].

For this work, the antisense oligonucleotide library $5^{\prime}$ gttatagttgtattc CAG TTT GTG TCC CAG GAT GTT GCC GTC TTC CTT GAA GTC GAT acctttcagctc 3' encoding amino acids I129-L142 of sgGFP was synthesized by COBARDE. This sequence corresponds to nucleotides 373-441 of sgGFP minus strand (equivalent to nucleotides $370-438$ in GFP). The codons subjected to random 
Table 2: Analysis of fluorescent and non-fluorescent mutants carrying single amino acid substitutions

\begin{tabular}{|c|c|c|c|c|c|c|c|c|c|c|c|c|c|c|c|c|c|c|c|c|c|c|}
\hline \multirow[t]{2}{*}{ WT aa } & \multirow[t]{2}{*}{ AS } & \multicolumn{9}{|c|}{ Hydrophobic aa } & \multicolumn{6}{|c|}{ Hydrophilic aa } & \multicolumn{3}{|c|}{ Acid } & \multicolumn{2}{|c|}{ Basic } & \multirow{2}{*}{$\begin{array}{l}\text { Stop } \\
\mathrm{X}\end{array}$} \\
\hline & & W & $\mathrm{F}$ & $\mathrm{P}$ & $M$ & $\mathrm{~L}$ & 1 & $\vee$ & $A$ & G & $N$ & Q & $S$ & $\mathrm{~T}$ & C & $Y$ & $\mathrm{D}$ & $\mathrm{E}$ & $\mathrm{R}$ & $\mathrm{K}$ & $\mathrm{H}$ & \\
\hline $\mathrm{FI3I}$ & B & & & I & & I & I & & $2 *$ & & & 1 & & 1 & & & 1 & & & 1 & 1 & \\
\hline $\mathrm{K} 132$ & $\mathrm{E}$ & I & & & & & I & & I & I & & & & & & & & I & 2 & & & \\
\hline EI33 & $\mathrm{E}$ & & & I & & 2 & & I & 3 & I & I & & I & I & & & I & & 3 & & & \\
\hline DI34 & $\mathrm{E}$ & & I & & & & & & & & & I & 2 & 2 & & & & & 2 & & & \\
\hline GI35 & B & & & & & 1 & $I^{*}$ & $I^{*}$ & & I & & & $I^{*}$ & & 1 & 1 & & & & & & \\
\hline $\mathrm{N} 136$ & $E$ & & I & 4 & $\mathbf{I}$ & I & & 4 & 4 & & & $\mathbf{I}$ & & 2 & & & & & I & I & $\mathbf{I}$ & \\
\hline 1137 & B & & & & & 4 & & 2 & & I & & & $I^{*}$ & & & & 1 & 2 & 2 & & & I \\
\hline LI38 & B & & I & & 2 & 2 & I & & & $I^{*}$ & & & & & 1 & & & & 1 & & & \\
\hline GI39 & $E$ & & & I* & & 2 & & & I & I & I & 2 & 2 & I & I & & & & & 2 & $\mathbf{I}$ & \\
\hline $\mathrm{HI} 40$ & $\mathrm{E}$ & & & 1 & & & I* & & & & I & & & & & I & & & 2 & 2 & & \\
\hline $\mathrm{K}|4|$ & $E$ & & & $2 *$ & 1 & & & & I & & I & & & I & & & & & I & & I & \\
\hline LI 42 & B & I & & & & & I & I & & 2 & $I$ & & 1 & & & & 1 & & & & & \\
\hline
\end{tabular}

Mutants displaying a green-fluorescent phenotype to the naked eye are shown in bold numbers. Mutants displaying a white phenotype are shown in italic numbers. Mutants displaying a pale green-fluorescent phenotype are shown in underlined numbers. Those mutants labeled with asterisk turned pale green-fluorescent after prolonged growing at room temperature. The number represents the number of times that each mutant was found. In this table, empty spaces represent wild-type amino acids. B means buried amino acid; E, solvent exposed amino acid. During screening of fluorescent mutants, seven clones corresponded to wt sgGFP generated with the mixtures of degenerated oligonucleotides.

deletion are shown in capital letters and the non-modified flanking regions in lower case. This sequence also included six silent nucleotide substitutions (bold letters) that change $\mathrm{t} 376 \mathrm{c}, \mathrm{a} 378 \mathrm{~g}, \mathrm{t} 387 \mathrm{c}, \mathrm{t} 402 \mathrm{c}, \mathrm{t} 411 \mathrm{c}$ and $\mathrm{t} 424 \mathrm{c}$ in the coding strand. Such modifications were done to differentiate wild-type clones generated in the mutant library from wild-type clones obtained by re-ligation of the recipient plasmid and to reduce the $\mathrm{dA}$ ratio in the oligo. The Fmoc-Cl solution used to repeatedly block part of the growing oligo was $12 \mathrm{mM}$ which produces approximately $50 \%$ deletion mutants per site.

All other ancillary oligonucleotides used in this research were synthesized at the core facility of our Institute, as recommended by the DNA synthesizer manufacturer (Applied Biosystems, Inc.).

\section{Construction of the recipient plasmid pT4GFPMlu}

sgGFP is an engineered variant (F64L, S65C, I167T) of GFP, being brighter than wild-type GFP because of its greater solubility and folding at $37^{\circ} \mathrm{C}$, displaying unique excitation and emission peaks at $474 \mathrm{~nm}$ and $509 \mathrm{~nm}$ respectively [28]. sgGFP gene from vector pQBI25 (QBIOgene) was subjected to two site-directed mutagenesis steps to perform the silent nucleotide substitutions $\mathrm{t} 234 \mathrm{C}$ and $\mathrm{a} 327 \mathrm{~g}$, following the procedure published by Merino et al [29]. The first change destroys an Nde I restriction site found in the gene and the latter creates an Mlu I restriction site, with no alteration of amino acid sequence. The modified gene was amplified with two external primers containing Nde I and Xho I restriction sites and was cloned into a pT4 cloning vector [30] under control of the trc promoter. This construction was finally digested with the restriction enzymes Mlu I and Acc I (New England Biolabs) to prepare the recipient cloning vector pT4GFPMlu.

\section{Mutagenesis, cloning and selection of GFPs carrying random amino acid deletions}

Mutant cassettes were generated by extension of the primers GFP-MluI (5'ctacaagacgcgtgctgaagtcaagtttgaaggtgatacccttgttaatagaatcgagctgaaaggt $3^{\prime}$ ) and GFP-AccI (5'tgccatgatgtatacattgtgtgagttatagttgtattc 3 '), using the oligonucleotide library as template (Figure 2); these primers contain the Mlu I and Acc I restriction sites, respectively, for cloning purposes. $50 \mathrm{pmol}$ of each primer and $5 \mathrm{pmol}$ of the oligonucleotide library were subjected to PCR. TaqGold polymerase (Applied Biosystems) was used for the PCR, following the conditions: $1 \times: 95^{\circ} \mathrm{C}$ for $5 \mathrm{~min}$ and 15X: $94^{\circ} \mathrm{C}$ for $30 \mathrm{sec}, 30^{\circ} \mathrm{C}$ for $30 \mathrm{sec}, 72^{\circ} \mathrm{C}$ for $30 \mathrm{sec}$.

The extended fragment was purified by agarose gel and digested with Mlu I and Acc I restriction enzymes. The digested product was ligated overnight to $3 \mathrm{pmol}$ of the recipient plasmid pT4GFPMlu using T4 DNA ligase (New Englad Biolabs). The ligation mixture was electroporated into XL1-Blue cells. A 1/1000 aliquot of the transformants was plated on a kanamycin-containing LB plate, incubating for $24 \mathrm{~h}$ at $37^{\circ} \mathrm{C}$ to quantify the library size. The remaining transformants were grown overnight into 20 $\mathrm{ml}$ of kanamycin-containing LB to recover the transformants as a library of plasmids. 
The pool of plasmids was re-electroporated into XL1-Blue cells and the transformants were grown on kanamycincontaining LB plates at $37^{\circ} \mathrm{C}$ for $24 \mathrm{~h} .40$ colonies displaying a green-fluorescent phenotype to the naked eye were randomly chosen and sequenced. 33 white colonies were also randomly chosen and sequenced to analyze the diversity of mutations generated in the library, as well as the type of deletions that destroy fluorescence.

Amino acid deletions created by site-directed mutagenesis The site-directed mutants sgGFP- $\Delta$ F131， sgGFP- $\Delta$ K132, sgGFP- $\triangle$ E133， sgGFP- $\Delta$ D134， sgGFP- $\Delta$ G135， sgGFP$\Delta$ N136， sgGFP- $\Delta$ I137， sgGFP- $\Delta$ L138， sgGFP- $\Delta$ G139， sgGFP- $\Delta$ H140 and sgGFP- $\Delta$ L142, which delete one amino acid each, as indicated in their names, were constructed following the standard protocol described for the library of GFPs carrying random amino acid deletions, replacing the oligonucleotide library by each of the specific primers shown on Figure 2 . The double mutant sgGFP$\Delta \mathrm{I} 129: \Delta \mathrm{D} 130$ that combines deletion of I129 and D130 was also assembled. Each mutant was confirmed by DNA sequencing.

\section{Random single amino acid substitutions generated by site- directed mutagenesis}

Single substitution of residues F131, K132, E133, D134, G135, N136, I137, L138, G139, H140, K140 and L142 with any of the other amino acids (aa) was performed by the twelve independently synthesized degenerated oligonucleotides shown on Figure 2. These primers were grouped into three sets of four oligonucleotides and cloned as described above for the mutagenic oligonucleotide. Several colonies from each set, displaying a green fluorescent phenotype, pale-green phenotype or white phenotype to the naked eye after 24 hours of growing at $37^{\circ} \mathrm{C}$, were randomly chosen and analyzed by DNA sequencing.

\section{Immunoblotting and fluorescence analysis of sgGFPs}

XL1-Blue cells expressing the appropriate sgGFP protein (either wild-type or mutant) were inoculated into kanamycin-supplemented liquid LB and the cultures were grown overnight, under shaking at $37^{\circ} \mathrm{C}$. Whole cell extracts were prepared from the cultures normalized at the same optical density $\left(\mathrm{OD}_{600 \mathrm{~nm}}\right)$. The pellet was obtained by centrifugation and resuspended in a B-PER (nonionic detergent in $20 \mathrm{mM}$ Tris-HCl, pH 7.5) bacterial protein extraction reagent (PIERCE). A protease inhibitor cocktail, complete EDTA-free (Roche), was added as recommended. Insoluble and soluble protein fractions were obtained by centrifugation. Total protein concentration was measured by Bradford reagent (BIORAD). For Western blotting analysis, $20 \mu \mathrm{g}$ of proteins of the soluble and insoluble fractions were resolved by $12 \%$ SDS-PAGE and then transferred to nitrocellulose membranes (Amersham
Pharmacia Bioscience) for 1 hour at $70 \mathrm{~mA}$ in a semi-dry transfer unit (Hoefer SemiPhor-Amersham Pharmacia Biotech). Western blotting was performed following standard protocols [31], using anti-GFP (Clontech) for the detection.

The fluorescence assay was recorded on a Perkin Elmer Luminescence Spectrometer LS50B or a Genesis workstation TECAN using the module safire. All measurements were made in triplicate.

For determination of the quantum yield of mutants sgGFP- $\Delta \mathrm{I} 129$ and sgGFP- $\Delta \mathrm{D} 130$, a calibration curve of absorbance at $474 \mathrm{~nm}$ versus fluorescence emission at 508 $\mathrm{nm}$ was performed for lysates of wild-type sgGFP, using the luminescence spectrometer LS50B (Perkin Elmer). Quantum yield of the active mutants carrying deletions, relative to wild-type sgGFP, was calculated by determining the absorbance and fluorescence of cell extracts of one sample and extrapolating to the calibration curve. All measurements were made in triplicate.

\section{Authors' contributions}

GFR, MR, JO and AMP carried out the mutagenesis studies. XS participated in the design of the study and drafted part of the manuscript. PG conceived of the study, and participated in its design and coordination and drafted most of the manuscript. All authors read and approved the final manuscript.

\section{Acknowledgements}

J.O. acknowledges financial support from CONACYT (grant 50952). We thank Eugenio López, Santiago Becerra, Soledad Juárez, Leopoldo Guereca and Jorge A. Yáñez for excellent technical assistance. We are also indebted to Dr. Anne-Laure Chauvin and Dr. Hugh Mackie for careful revision of the manuscript.

\section{References}

I. Shimomura O: Structure of the chromophore of aequorea green fluorescent protein. FEBS Lett 1979, 104:220-2.

2. Prasher DC, Eckenrode VK, Ward WW, Prendergast FG, Cormier MJ: Primary structure of the Aequorea victoria green-fluorescent protein. Gene 1992, I I I:229-33.

3. Chalfie M, Tu Y, Euskirchen G, Ward WW, Prasher DC: Green fluorescent protein as a marker for gene expression. Science 1994, 263:802-5.

4. Cormack BP, Valdivia RH, Falkow S: FACS-optimized mutants of the green fluorescent protein (GFP). Gene 1996, I73:33-8.

5. Heim R, Prasher DC, Tsien RY: Wavelength mutations and posttranslational autoxidation of green fluorescent protein. Proc Natl Acad Sci USA 1994, 91:12501-4.

6. Davis SJ, Vierstra RD: Soluble, highly fluorescent variants of green fluorescent protein (GFP) for use in higher plants. Plant Mol Biol 1998, 36:521-8.

7. Crameri A, Whitehorn EA, Tate E, Stemmer WP: Improved green fluorescent protein by molecular evolution using DNA shuffling. Nat Biotechnol 1996, I 4:315-9.

8. Bokman SH, Ward WW: Renaturation of Aequorea gree-fluorescent protein. Biochem Biophys Res Commun I 981, I 01:1372-80.

9. Verkhusha VV, Kuznetsova IM, Stepanenko OV, Zaraisky AG, Shavlovsky MM, Turoverov KK, Uversky VN: High stability of Discosoma DsRed as compared to Aequorea EGFP. Biochemistry 2003, 42:7879-84. 
10. Li X, Zhang G, Ngo N, Zhao X, Kain SR, Huang CC: Deletions of the Aequorea victoria green fluorescent protein define the minimal domain required for fluorescence. J Biol Chem 1997, 272:28545-9.

II. Kitamura K, Yoshida C, Nishigaki K: GFPs of insertion mutation generated by molecular size-altering block shuffling. FEBS Lett 2003, 555:483-8.

12. Abedi MR, Caponigro G, Kamb A: Green fluorescent protein as a scaffold for intracellular presentation of peptides. Nucleic Acids Res 1998, 26:623-30.

13. Baird GS, Zacharias DA, Tsien RY: Circular permutation and receptor insertion within green fluorescent proteins. Proc Natl Acad Sci USA 1999, 96: I 124I-6.

14. Osuna J, Yanez J, Soberon X, Gaytan P: Protein evolution by codon-based random deletions. Nucleic Acids Res 2004, 32:el 36.

15. Banerjee S, Pieper U, Kapadia G, Pannell LK, Herzberg O: Role of the omega-loop in the activity, substrate specificity, and structure of class A beta-lactamase. Biochemistry 1998, 37:3286-96.

16. Gurskaya NG, Savitsky AP, Yanushevich YG, Lukyanov SA, Lukyanov $\mathrm{KA}$ : Color transitions in coral's fluorescent proteins by sitedirected mutagenesis. BMC Biochem 200I, 2:6.

17. Stepanenko OV, Verkhusha VV, Kazakov VI, Shavlovsky MM, Kuznetsova IM, Uversky VN, Turoverov KK: Comparative studies on the structure and stability of fluorescent proteins EGFP, zFP506, mRFPI, "dimer2", and DsRed I. Biochemistry 2004, 43: | 49|3-23.

18. Young L, Dong Q: Two-step total gene synthesis method. Nucleic Acids Res 2004, 32:e59.

19. Jayaraman K, Fingar SA, Shah J, Fyles J: Polymerase chain reactionmediated gene synthesis: synthesis of a gene coding for isozyme $c$ of horseradish peroxidase. Proc Natl Acad Sci USA I99I, 88:4084-8.

20. Binkowski BF, Richmond KE, Kaysen J, Sussman MR, Belshaw PJ: Correcting errors in synthetic DNA through consensus shuffling. Nucleic Acids Res 2005, 33:e55.

21. Glen Research: [http://www.glenres.com].

22. Bosley $A D$, Ostermeier M: Mathematical expressions useful in the construction, description and evaluation of protein libraries. Biomol Eng 2005, 22:57-6I.

23. Gurskaya NG, Verkhusha VV, Shcheglov AS, Staroverov DB, Chepurnykh TV, Fradkov AF, Lukyanov S, Lukyanov KA: Engineering of a monomeric green-to-red photoactivatable fluorescent protein induced by blue light. Nat Biotechnol 2006, 24:46I-5.

24. Quillin ML, Anstrom DM, Shu X, O'Leary S, Kallio K, Chudakov DM, Remington S): Kindling fluorescent protein from Anemonia sulcata: dark-state structure at 1.38 A resolution. Biochemistry 2005, 44:5774-87.

25. DePristo MA, Weinreich DM, Hartl DL: Missense meanderings in sequence space: a biophysical view of protein evolution. Nat Rev Genet 2005, 6:678-87.

26. Gurskaya NG, Fradkov AF, Pounkova NI, Staroverov DB, Bulina ME, Yanushevich YG, Labas YA, Lukyanov S, Lukyanov KA: A colourless green fluorescent protein homologue from the non-fluorescent hydromedusa Aequorea coerulescens and its fluorescent mutants. Biochem J 2003, 373:403-8.

27. Bajaj K, Chakrabarti P, Varadarajan R: Mutagenesis-based definitions and probes of residue burial in proteins. Proc Natl Acad Sci USA 2005, 102:16221-6.

28. Q-BIOgene [http://www.qbiogene.com/products/gene-expression/ superGLOI.shtml]

29. Merino E, Osuna J, Bolivar F, Soberon X: A general, PCR-based method for single or combinatorial oligonucleotide-directed mutagenesis on pUC/MI3 vectors. Biotechniques 1992, 1 2:508-10.

30. Gaytan P, Osuna J, Soberon X: Novel ceftazidime-resistance beta-lactamases generated by a codon-based mutagenesis method and selection. Nucleic Acids Res 2002, 30:e84.

31. Sambrook J, Fritsch EF, Maniatis T: Molecular cloning: a laboratory manual. 2nd edition. New York: Cold Spring Harbor Laboratory Press; 1989.

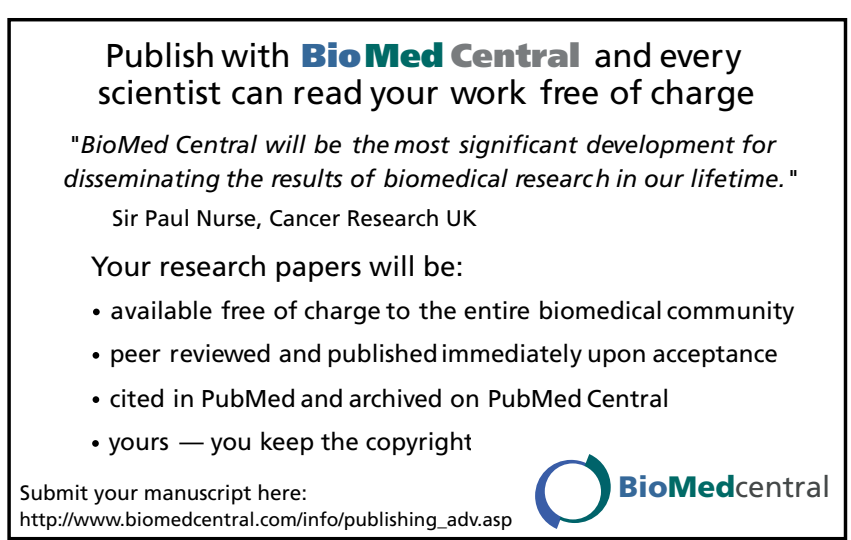

